\title{
GLOBALIZACIÓN E \\ INDUSTRIAS CULTURALES
}

\section{Alejandro Sánchez López de Mesa}

\section{CUANDO DESDE LA ISLA SEATISBAN BARCOS ${ }^{1} \ldots$. A LO LEJOS.}

Este escrito se cuece a la sombra de la pregunta por la relación entre las artes y la democracia. Pregunta que organiza el debate sobre las políticas de comunicación en América Latina a partir de los noventa, al que este escrito hace un aporte puntual. Nos aproximamos a las artes en tanto bienes simbólicos, para dar cuenta de un rango amplio de productos y productores, entre los que se incluyen los artículos mediáticos masivos y la gama diversa de "creaciones del espíritu" que circulan por internet. Nos interesan en tanto escenarios en los que se construyen representaciones y sentidos; donde se ponen en escena maneras de ver y entender el mundo; categorías, conceptos, lenguajes que utilizamos para hacer inteligible la sociedad en que vivimos.

Pese a que en Colombia el debate ciudadano sobre las industrias culturales es pre-

\section{RESUMEN}

El artículo encara la pregunta por el impacto de la globalización sobre la producción, distribución, promoción y consumo de bienes culturales. Antes de examinarla en su complejísima amplitud, el autor propone un hilo conductor que vincula tres problemáticas: la necesidad de cuestionar los conceptos de identidad y cultura presentes en interpretaciones comunes sobre la globalización; un sucinto repaso de las características del proceso de concentración en las industrias culturales; y una valoración de las potenciales repercusiones de las obligaciones más controvertidas que en materia de propiedad intelectual se negocia en los foros multilaterales, los acuerdos regionales y los tratados bilaterales de libre comercio.

\section{ABSTRACT}

The article raises the question concerning the impact of globalization on the production, distribution, promotion and consumption of cultural goods. Instead of tackling the question from its complex perspective, the author proposes a thread that involves three problems: The need for questioning the concepts of identity and culture which are present in frequent interpretations of globalization, a concise review of the characteristics of the concentration process in cultural industries, and an assessment of the potential effects of one of the most controversial issues in the matter of copyright, which is negotiated in multilateral forums, regional agreements and bilateral free trade treaties.

\section{PALABRAS CLAVE}

Globalización, cultura, industrias culturales, derechos de autor (copyright), propiedad intelectual.

1 El enunciado es el título del capítulo del texto Balsas y Medusas de Germán Rey, en el que se analizan las repercusiones del tránsito del sistema mixto a la privatización de la televisión colombiana. REY, Germán (1998). Balsas y Medusas. En: Visibilidad comunicativa y narrativas politicas. Bogotá: CEREC, Fundación Social, FESCOL. 
cario, la reciente discusión sobre las cuotas de pantalla reservadas a la producción televisiva nacional en el marco de la negociación del Tratado de Libre Comercio con los Estados Unidos, puede permitir posicionar algunos asuntos en la agenda pública. La visibilidad lograda por la frágil coalición coyuntural de los canales, un movimiento de artistas, algunas personalidades académicas y funcionarios del ejecutivo, debe permitirnos llevar a la agenda pública preguntas por el desarrollo de la globalización cultural y sus consecuencias.

El campo de producción de bienes culturales experimenta en la actualidad transformaciones significativas. Examinarlas obliga a revisar los conceptos de cultura e identidad en algunos de los discursos sobre la globalización que suelen predominar en distintos lugares sociales de enunciación. Discursos que suelen hacer énfasis en los riesgos que la globalización supone para la "integridad cultural" y las identidades de los pueblos alrededor del mundo. Discursos que al orientar nuestra mirada hacia la urgencia de proteger espejismos identitarios nacionales, nos impiden apreciar el complejo proceso a través del cual la cultura se convierte en mercancía. Discursos que usan la globalización como una suerte

\section{RESEÑA DE AUTOR}

Alejandro Sánchez López de Mesa es Politólogo de la Universidad de los Andes, Magíster en estudios políticos del IEPRI de la Universidad Nacional de Colombia y profesor de la Facultad de Comunicación Social-Periodismo del Politécnico Grancolombiano. de mantra, tras el que suelen aguardar intereses políticos.

Como lo señala John Tomlinson, estos discursos "tienden a ver la globalización como una extensión-incluso como un eufemismo-del imperialismo cultural occidental"2. Tal comprensión se hallaría anclada en una particular manera de comprender la identidad como una posesión individual o colectiva en riesgo, una esencia particular inscrita en la memoria, los valores, creencias y prácticas de comunidades específicas. Tomlinson recuerda entonces que la identidad nacional es un producto de la modernidad, un mecanismo para organizar la experiencia cultural y fijarla a un territorio ${ }^{3}$.

Con la articulación progresiva de circuitos comunicativos y de información alrededor del planeta nuestra experiencia cultural atraviesa flujos comunicativos transnacionales. Prácticas, consumos, discursos son incorporados a nuestra cotidianidad por medio de un proceso complejo de apropiación y localización, que suelen desconocer las lecturas que hacen énfasis en el carácter destructivo de la globalización. Mientras pocos experimentan la "globalidad" en tanto experiencia de vivir y actuar más allá de las fronteras, vivencia diaria de un conjunto de relaciones sociales articuladas a partir de espacios sociales que trascienden el Estado nacional ${ }^{4}$, las mayorías experimentan procesos interpretativos de localización que necesariamente suponen apropiaciones y resignificaciones.

2 TOMLINSON, John (1999). Globalization and culture. Cambridge : Polity Press.

3 TOMLINSON, John (2003). Globalization and Cultural Identity. En: HELD, David y McGrew, Anthony. The Global Transformations Readers. An introduction to the globalization debate. Cambridge: Polity Press, Blackwell Publishing. p. 269-277.

4 BECK, Ulrico (1998). ¿Qué es la Globalización? Falacias del globalismo; Respuestas a la Globalización. España: Paidós. P. 128 Para Beck, "la sociedad mundial sería entonces un horizonte mundial (de sentido) que solo se abre si se mantiene como real en la comunicación". 
Las culturas, esas urdimbres de sentidos de los que habla Geertz, antes que desaparecer, son los insumos con los cuales nos valemos para relocalizar lo que nos llega de fuera. Como bien lo señala John B. Thompson, la recepción supone un proceso de negociación del contenido simbólico de los mensajes, "es un proceso social complejo en el que los individuos dan sentido activo a los mensajes, utilizándolos de manera distinta en el transcurso de sus vidas"s. Aquellos discursos que asimilan globalización al inestable concepto de "imperialismo occidental", no sólo desconocen el carácter hermenéutico de la recepción, sino que asumen erróneamente que basta con caracterizar la estructura mediática transnacional para suponer sus efectos sobre las culturas o la identidad.

Por supuesto, el proceso de localización de los flujos comunicativos es un proceso tenso, que ocurre en una coyuntura específica. Asistimos a un período en el que el Estado, instancia homogeneizadora que funda la nación, es cada vez menos capaz de controlar los flujos de conocimiento, información y capital. La globalización no sólo debilita al aparato burocrático, sino que además, a juicio de autores como Ulrich Beck, ha hecho que entre en crisis la matriz estadocéntrica que utilizábamos para entender la realidad ${ }^{6}$.

Al mismo tiempo, posiciones de sujeto, identidades distintas a la nacional cobran un mayor protagonismo en la cotidianidad de muchos individuos, al organizar su experiencia inmediata. Algunas de estas nuevas identidades, si bien son recreadas en rituales globales e instituciones transnacionales productoras de discurso, son re-creadas localmente, como lo demuestra el estudio de Martha Marín y Germán Muñoz sobre las culturas juveniles asociadas al Punk, el Heavy Metal o el Hip Hop ${ }^{7}$. La identidad nacional no desaparece, si bien debe convivir con otras pertenencias a otras comunidades imaginadas ${ }^{8}$.

Esta “inestabilidad representativa” de la que habla Gustavo Lins Ribeiro", se acentúa aún más en tanto algunas de esas diversas posiciones de sujeto, identidades con las que operamos en la vida diaria, suelen construirse desde prácticas de consumo. Identidades que ya no se definen por esencias ahistóricas como la etnia o la nación, sino que dependen de lo que uno posee o es capaz de apropiarse ${ }^{10}$. Identidades que se fundan en rituales de copresencia que son puestos en escena a través de las redes mediáticas transnacionales.

5 THOMPSON, John B. (1998). Los Media y la Modernidad. Una Teoría de los Medios de Comunicación. Barcelona: Paidós. P.228.

6 Así, esta supone que "el Estado y la sociedad son pensados, organizados y vividos de manera coincidente. El Estado territorial se convierte en contenedor de la sociedad”. Op. Cit. 4. P. 99.

7 MARÍN, Martha y MUÑOZ, Germán (2000). Secretos de mutantes. Música y creación en las culturas juveniles. Bogotá: Siglo del Hombre Editores, Universidad Central - DIUC.

8 De hecho, autores como Manuel Castells insisten en que la globalización, antes que destruir identidades, ha promivido el surgimiento de abundantes pertenencias desde las que se resalta a la globalización misma. Véase: CASTELLS, Manuel. “Globalización, sociedad y política en la Era de la Información”. En: Revista Análisis Político No. 37, mayo-agosto de 1999. IEPRI, Universidad Nacional.

9 LINS RIBEIRO, Gustavo (2003). Postimperialismo. Cultura y política en el mundo contemporáneo. Barcelona: Gedisa Editores.

10 GARCÍA CANCLINI, Néstor (1995). Consumidores y ciudadanos. Conflictos culturales de la globalización. México: Editorial Grijalbo. 
La inestabilidad representativa, los conflictos de identidad y la crisis de conceptos y del aparato burocrático estatal no suponen, sin embargo, la destrucción de culturas e identidades en la que aún insisten ciertos discursos sobre la globalización. Revisar los conceptos de cultura e identidad desde los que analizan la globalización los comunicadores y las facultades de comunicación resulta imperativo. Insistimos en que la urgencia por asumir la defensa de espejismos identitarios nacionales, nos impide analizar las' verdaderas repercusiones del complejo proceso a través del cual la cultura se convierte en mercancía.

La progresiva integración de las artes a los circuitos industriales de producción, circulación y consumo de bienes y servicios ha venido acompañada por una multiplicación impresionante de los canales y de los flujos. Al tiempo que se consolida un férreo control oligopólico de las redes y canales de distribución, aparecen nuevos ámbitos creativos y lugares, discursos y retóricas desde donde intenta fundarse la resistencia. Uno de estos últimos, según sus entusiastas, el universo blogger.

La globalización en el campo cultural, como en otros campos, produce nuevas formas de centralización de los procesos de creación, circulación y consumo en organizaciones cuya gestión cotidiana y procesos productivos, se descentralizan. Como lo señala Saskia Sassen ${ }^{11}$ en su estudio sobre el lugar de Nueva York, Londres y Tokio en el mundo en globalización, se configuran nuevos sistemas de coordinación (propios de sociedades postindustriales) a partir del desarrollo de polos de control particulares. Los derechos de propiedad sobre los bienes simbólicos y el conocimiento, y la capacidad de administrar las redes por donde circulan flujos comunicativos y de información son los elementos a partir de los cuales se produce esta progresiva centralización.

El mercado mediático global se encuentra altamente concentrado. Tal y como lo señala Robert W. McChesney, la desregulación y la privatización de sistemas de medios en el mundo han propiciado el desarrollo de megacorporaciones que controlan gigantescas redes de distribución y producción que se extienden por todo el planeta ${ }^{12}$. El mercado global planetario es oligopólico y es dominado por ocho transnacionales norteamericanas (Disney, AT\&T, Sony, General Electric, News Corporation, Seagram, Time Warner, Viacom) ${ }^{13}$ y el emporio alemán Bertelsman. Estos son los nodos donde se articula y centraliza la red.

Como lo señalan McChesney y otros autores, existe una muy baja integración horizontal entre estas empresas. Es decir, suelen controlar sólo sectores específicos de la industria mediática. Sin embargo, sî existe una importante integración vertical, lo que supone que las mismas empresas son dueñas tanto del contenido de los transformations readers. An introduction to the globalization debate. Cambridge: Polity Press, Blackwell publishing. P. 360-368.

13 Como lo señalan varios autores, el predominio de las industrias mediáticas norteamericanas es, en parte, el resultado de las ventajas que supone el enorme tamaño del mercado de ese país. Asimismo hay que señalar que tras la Segunda Guerra Mundial el gobierno de EE.UU. promovió la formación de un cártel (The Motion Picture Export Association) que favoreció la concentración en la industria del cine, al tiempo que exigió a los países beneficiarios del Plan Marshall la compra de determinadas cantidades de películas estadounidenses. 
bienes culturales, como de los canales a través de los cuales este se distribuye. Se trata de empresas altamente internacionalizadas, que según datos presentados por McChesney, para 1999 obtenían entre el 21 y el $73 \%$ de sus ganancias en mercados externos a su país de origen.

Las megacorporaciones se enlazan con una red compleja de emporios mediáticos de carácter regional o nacional a través de un rango diverso de alianzas. Empresas como Globo, Televisa, el grupo Clarín en Argentina, el grupo Cisneros en Venezuela o Mediaset (de propiedad de Silvio Berlusconi) suelen acoplarse progresivamente a las reglas de juego impuestas por los centros de la red, expandiéndose hacia mercados marginales o adaptando su producción a las necesidades de estos.

Para McChesney, las megacorporaciones mediáticas tienden a convertirse en un cártel en tanto suelen preferir aliarse en proyectos de riesgo compartido antes que competir. La competencia, dirá este autor, suele darse cada vez con más frecuencia en las megacorporaciones, antes que entre estas ${ }^{14}$. A su juicio, como al de Loost Smiers ${ }^{15}$, su tamaño y éxito supone un aspecto conservador que se manifiesta en un control estricto de los mensajes que circulan por sus redes mediáticas, discusión en la que no profundizaremos aquí.

Al tiempo que se acrecienta esta centralización del espacio mediático, las decisiones que determinan la estructura del campo cultural son tomadas cada vez más por tecnocracias en escenarios especializados que eluden los lugares desde los que tra- dicionalmente se pensó lo cultural. Como lo señala Joost Smiers, la reflexión sobre la cultura y las implicaciones de las transformaciones actuales para el campo cultural ha cambiado de manos.

Hasta 1984 fue la Unesco el foro donde se desarrollaron las discusiones sobre la transnacionalización de las industrias culturales. De allí surgieron notorios diagnósticos y propuestas de política pública, como aquellos contenidos en el Informe Macbride, publicado a mediados de los setenta, que alentó el diseño de políticas de comunicación orientadas a corregir el desequilibrio informativo en América Latina. Pese a sus ambiciosos, quizás utópicos propósitos y a la férrea oposición de los Estados Unidos, su poderosa industria cultural y algunos medios locales, este esfuerzo inspiró el desarrollo de agencias y redes informativas en el tercer mundo y dejó sentadas las bases de la discusión en torno al "derecho a la comunicación" que aún inspira reformas y reflexiones académicas.

Luego de que Estados Unidos se retirara de la Unesco en 1984, lo que ocasionó una reducción dramática de sus recursos, la reflexión multilateral acerca del impacto de la liberalización comercial sobre la producción de cultura, hubo de desplazarse a escenarios que le son extraños, como la Organización Mundial del Comercio (OMC), creada en 1994 para reemplazar al Acuerdo General sobre Comercio y Aranceles (GATT, por sus siglas en inglés). La OMC, cuya función es promover la liberalización progresiva del comercio en el mundo, resulta un foro técnico al que 
le son extraños los debates sobre los riesgos que supone dejar exclusivamente a las fuerzas del mercado las decisiones relativas a la producción, distribución, promoción y consumo de bienes simbólicos.

Acerca del funcionamiento de la OMC vale la pena hacer dos precisiones. Por un lado, la organización opera sobre la base de un principio de no discriminación que en la práctica se concreta en dos disposiciones: el principio de nación más favorecida (NMF) y el de trato nacional (TN). En virtud del primero, las concesiones comerciales otorgadas a cualquier país bajo el amparo de la normatividad de la OMC deberán extenderse también a los demás miembros. El segundo establece que se debe dar el mismo tratamiento a oferentes nacionales y extranjeros.

Por otro lado, es útil recordar que la OMC cuenta con un tribunal para resolver disputas comerciales internacionales. El tribunal de arbitramento opera como un sistema de justicia privada con la autoridad para disciplinar a aquellos estados cuyas normas atenten contra los términos del acuerdo. El tribunal está compuesto por tres oficiales designados -no elegidos- que se reúne en secreto, sin mayor cubrimiento mediático o participación ciudadana. Mientras las decisiones son de obligatorio cumplimiento, apelar es prácticamente imposible.

La liberalización del comercio de bienes culturales no ha tenido un curso fácil en el seno de la OMC. En principio, la oposición europea liderada por Francia y preocupada por el predominio de las multinacionales norteamericanas en el universo mediático, logró el establecimiento de una amplia reserva cultural, que establece que los esta- dos miembros no están obligados a abrir sus mercados de bienes culturales y pueden proteger y promover sus mercados domésticos.

No olvidemos que la OMC pretende dar forma a un acuerdo de alcance orbital en el que se contemplan temas puramente comerciales (relativos a la eliminación de barreras al intercambio de bienes y servicios) y también disciplinas referidas a temas no propiamente comerciales como la propiedad intelectual, inversiones, medidas antidumping, solución de controversias, compras públicas, etc. La Cultura, elusiva como la que más, aparece y desaparece en distintos lugares, en varios de los temas en torno a los que se desarrolla la discusión.

Un repaso sucinto nos permite ver que es un tema puramente comercial cuando se discute la eliminación de las barreras arancelarias al intercambio de bienes y/o servicios en el campo cultural. También es tema del capítulo de inversiones cuando se discute la conveniencia de permitir o no la inversión extranjera en los medios, la industria publicitaria, las distribuidoras de productos culturales, los museos, las comercializadoras de arte, los proveedores de internet, entre otros. Un tema del capítulo de telecomunicaciones cuando se discuten las condiciones de acceso de las multinacionales a la infraestructura nacional instalada, que en Colombia es patrimonio público. Un tema de propiedad intelectual, en tanto allí se contemplan disposiciones relativas a los derechos de autor, de reproducción y de marcas, así como derechos sobre conocimientos ancestrales de comunidades indígenas o campesinas. Sobre este último tema habremos de volver luego.

Precisar el alcance de una "reserva cultural" no es por lo tanto nada fácil. De ahí 
que la $\mathrm{OMC}^{16} \tan$ sólo haya insistido en una cláusula general -una página en blancoque permite un amplio margen de maniobra a sus miembros. De hecho, la cumbre de 1998 que reunió en Ottawa a ministros de cultura de 20 naciones, para discutir la posibilidad de dar con un marco normativo común que les permitiera proteger sus industrias culturales, tan sólo concluyó con la recomendación de mantener la cultura fuera de la OMC. Si bien en el marco de la Ronda de Doha el tema se ha sometido a consideraciones, no se han logrado avances significativos.

En últimas, se trata de otro de los obstáculos que enfrenta la $\mathrm{OMC}$, similar a lo que ocurre en temas como agricultura, servicios, inversiones o propiedad intelectual a raíz de conflictos entre sus miembros más poderosos (Estados Unidos, Europa y Japón) o entre estos y agrupaciones de países del tercer mundo. Obstáculos que han propiciado un cambio en la estrategia liberalizadora de las potencias occidentales, que han optado por avanzar sus intereses estratégicos ya no en el gran foro multilateral, donde las decisiones han de tomarse por consenso, sino a través de negociaciones de carácter regional o bilateral. ${ }^{17}$

Un barrido poco sistemático, si bien amplio, de las discusiones sobre el tema cultural en los tratados de libre comercio nos permite concluir que la discusión suele centrarse en temas asociados a los capítulos de servicios e inversiones. Así, los temas que más preocupan a los interesados tienen que ver con asegurar que en las industrias culturales predominen capital e inversionistas locales; que se mantengan cuotas de exhibición para la producción local, que se mantengan prerrogativas especiales para los profesionales nacionales frente a los extranjeros y que se mantenga la potestad del Estado para financiar o apoyar la producción local de bienes culturales.

Por lo general, esto supone incluir las listas de sectores, medidas o disposiciones que no serían contempladas por las obligaciones del acuerdo, en los anexos de medidas disconformes que contemple el tratado. En estos términos, por ejemplo, parece que terminará saldándose el cuestionamiento a la estrategia negociadora que planteara la Coalición para la Diversidad Cultural en el marco de las negociaciones del TLC con Estados Unidos. De cierto modo, esta resulta una solución respaldada por la incapacidad de la comunidad internacional para tratar de manera más sistemática el tema cultural en la OMC.

Los temas de propiedad intelectual también suscitan intensas discusiones, que, sin embargo, no suelen atraer tanta atención en el campo de producción de bienes culturales. Vale aclarar que el sistema internacional para la protección de los derechos de propiedad intelectual es sumamente complejo, pues coexisten acuerdos multilaterales (como aquellos suscritos en el ámbito de la OMC), convenciones no obligatorias (como la Convención sobre Diversidad Biológica, CBD), foros permanentes de discusión

16 Op. Cit. 12.p. 263-264.

17 Así, uno de los interes de Estados Unidos al negociar tratados de libre comercio en años recientes es dar forma a alianzas que le permitan avanzar reformas en la OMC en áreas estratégicas como propiedad intelectual, inversiones, servicios y compras públicas, entre otras. Véase: SCHOTT, Jeffrey J. (Ed) (2004). Free Trade Agreements. US Strategies and Priorities. Washington D.C.: Institute for International Economics. P. 13. 
(como la Organización Mundial de Propiedad Intelectual, WIPO), acuerdos regionales y bilaterales.

En su núcleo se encuentra el Acuerdo sobre Derechos de Propiedad Intelectual en el Comercio (ADPIC, o TRIP por sus siglas en inglés). El ADPIC fue adoptado en 1986 a través de la Declaración de Punta del Este, durante la ronda del GATT celebrada en Uruguay. Según autores como Graham Dutfield $^{18}$, es claro que los países subdesarrollados lo aceptaron en tanto este representaba un acuerdo de mínimos, que les permitiría evitar las sanciones unilaterales con países desarrollados (especialmente Estados Unidos) que amenazaban a aquellos que no implementaran políticas para la protección de la propiedad intelectual. Su aprobación también estuvo condicionada a concesiones mínimas en áreas como agricultura y textiles.

Sin duda, la producción, distribución, promoción y consumo de bienes culturales pueden verse afectados por un rango amplísimo de las disposiciones, derechos y obligaciones contemplados en el ADPIC o en cualquier capítulo de propiedad intelectual de un tratado de libre comercio. Existe, por ejemplo, una abundante bibliografía al respecto del impacto de los ADPIC sobre la diversidad biológica y cultural, especialmente en lo relativo a los derechos de propiedad sobre seres vivos y la preservación del conocimiento tradicional de comunidades indígenas y campesinas. Aquí, sin embargo, se realiza una aproximación pre- liminar a otra de las obligaciones allí consagradas, el derecho de reproducción de bienes culturales o derecho de autor.

Con el copyright o derecho exclusivo de reproducción, como con los demás derechos de propiedad intelectual, se busca resolver una falla en el mercado del conocimiento y las creaciones artísticas. En tanto las invenciones y las creaciones son costosas de producir y fáciles de copiar, no existirían estímulos para su producción o su puesta a disposición del público. Con estos instrumentos se busca equilibrar el reconocimiento de la inversión hecha por creadores, inventores y artistas, quienes por su parte se comprometen a hacer públicas sus creaciones y permitir a otros beneficiarse de estas.

Así, lo que ampara el establecimiento del derecho de autor es un bien público: crear artificialmente un equilibrio entre el interés de los creadores y el de la sociedad que habrá de disfrutar los bienes. Recientemente, sin embargo, desde la academia y algunos lugares virtuales de producción de discurso, este principio ha empezado a ser cuestionado. El argumento soporte de las críticas no tiene aristas simples, especialmente en tanto que quienes lo proponen se enfrentan a uno de los cánones que soporta el funcionamiento del capitalismo.

Para estos críticos la concentración mediática impide que el derecho de autor cumpla su cometido, pues antes que hacer viable un mercado plural de bienes culturales, sólo refuerza la tendencia anticom-

$18 \mathrm{Al}$ respecto resulta ilustrativo destacar la obra de Graham Dutfield, quien por muchos años ha sido director académico del programa de la UNCTAD K sobre propiedad intelectual, Senior Research Asóciate del Oxford Centre for Intellectual Property Research y es uno de los autores más renombrados sobre este tema. De sus muchas obras cabe destacar: Posey, D.A. and Dutfiels, G. (1996). Beyond Intellectual Property: Towards Traditional Resource Rights for Indigenous Peoples and Local Communities. Ottawa: International Development Research Centre. 
petitiva que caracteriza al mercado. De hecho, autores como Smiers ${ }^{19}$ encuentran contradictorio que los derechos monopólicos para copiar y distribuir los bienes culturales se le concedan a una entidad que no es ni creadora, ni autora, sino que simplemente se dedica a comercializar los bienes que adquiere. Como lo sugirió en 1996 la Comisión Internacional de las Naciones Unidas sobre Cultura y Desarrollo en su informe a la asamblea general ${ }^{20}$, los ADPIC han alejado los derechos de autor para hacerlos operar a partir de lógicas puramente comerciales.

Las megacorporaciones que controlan las redes de distribución de bienes culturales, han de nutrirlas permanentemente de contenidos que han de renovarse al ritmo de las preferencias de las audiencias. El control de los contenidos que asegura el derecho de autor refuerza su posición hegemónica. En el futuro, es seguro que la discusión sobre el control de los contenidos que circulan por internet habrá de cobrar protagonismo. Podemos suponer que nuevas tecnologías y normas cada vez más ambiciosas permitirán exigir el pago de regalías no sólo por la multiplicación y distribución de los contenidos, sino quizás también por cada consumo individual de estos.

El control de los bienes simbólicos es además un tema central para Estados Unidos en el marco de las negociaciones comerciales. Como lo señala Smiers, sus embajadas ahora monitorean sistemáti- camente las violaciones del derecho de marcas y el derecho de autor. La legislación norteamericana ha establecido listas de espera y sanciones contra aquellos países que no demuestren resultados concretos en la lucha contra la piratería. Sanciones que, en el caso del TLC que negociaron Estados Unidos y Singapore, obligaron a este último a imponer una de las legislaciones más estrictas del mundo en esta materia. El cabildeo corporativo también juega un papel importante.

No olvidemos que si bien el andamiaje institucional con el que cuenta la OMC para enfrentar la violación del derecho de autor en el mundo es limitado, no ocurre lo mismo en los tratados bilaterales de libre comercio. Si los tratados Sur-sur sufren de una incapacidad estructural para institucionalizarse, en los TLC que se negocian entre países desarrollados y países subdesarrollados, suele ocurrir que la voluntad y los recursos (de todo tipo) del país del norte, suplen cualquier debilidad institucional del acuerdo con miras a obligar el cumplimiento de las obligaciones pactadas.

A los detractores del actual sistema de derechos de autor les preocupa igualmente una eventual reducción del "dominio o el ámbito de la cultura pública”. Señalan con acierto que toda creación suele estar en deuda con una cultura pública, con un conjunto de sentidos, de símbolos, de signos que hacen parte de un acumulado intergeneracional que el derecho de autor

19 Joost Smiers, profesor de Ciencia Política de las Artes en la Universidad de Utrech, en Holanda, y profesor visitante en otras universidades del primer mundo es uno de los académicos que de manera más prolífica ha estudiado el tema de las contradicciones inherentes al funcionamiento del copyright. Sus obras presentan alternativas de política pública con miras a hacerle frente a estas contradicciones. Véase, además de la obra aquí citada: JOOST, Smiers. The Abolition of Copyright: Better for Artist, Third World Countries and the Public Domain. En: TOWSE, Ruth (2002). Copyright in the cultural industries. London: Cheltenham.

20 PÉREZ DE CUELLAR, Javier (1996). Our creative diversity. Report of the World Commission on Culture and Development. París: Unesco. 
desconoce. Acumulado que es la base de la que emergen las nuevas creaciones y donde cobran sentido.

El concepto mismo de originalidad, en el que se funda el derecho de autor, dirá Joost Smiers, es en la práctica mucho menos preciso y evidente de lo que parece a primera vista $^{21}$. Las fuentes, los recursos que el autor utiliza en su obra, suelen hacer parte de repertorios de insumos que conforman la herencia cultural común. "Es imposible reclamar originalidad absoluta. Esta es la razón por la que ni en los ADPIC ni en las leyes nacionales se define expresamente qué es una invención".

Por supuesto, el régimen de protección de la propiedad intelectual tiene problemas para reconocer el concepto de derechos colectivos de propiedad implícito en la idea de una herencia cultural común. Su fundamento es la idea misma de la apropiación privada-individual del conocimiento o las creaciones artísticas. Los entornos digitales, sin embargo, han embrollado aún más el problema. En entornos sobrecargados de flujos comunicativos y bienes simbólicos las creaciones se asemejan cada vez más a pastiches, a colages hechos de fragmentos rescatados en los circuitos multimediales.

¿Supone el actual sistema de derechos de autor la privatización de la herencia cultural común, del ámbito cultural público en el que se fundan las nuevas creaciones? ¿Supone hipotecar nuestra creatividad en el futuro? ¿Perdemos la posibilidad de controlar la producción local de bienes culturales; de hacer de esta un asunto público? La discusión supone un conflicto tan complejo como intenso.

La puja ya comenzó. La propuesta subversiva del Creative Commons -que gana adeptos y que permite la utilización de contenidos de internet en nuevas creaciones sin exigir ya el pago de regalías aunque sí un registro continuo de los usos que se hace de estos- representa un primer quiebre en el aparente consenso que sustenta el derecho de autor. Una visión novedosa de un orden alternativo aún en construcción. Más que hacer apuestas políticas, este escrito reconoce la centralidad e importancia de esta discusión en el debate sobre globalización y cultura e intenta una primera aproximación.

\section{BIBLIOGRAFÍA}

- BECK, Ulrico (1998). ¿Que es la globalización? Falacias del globalismo, Respuestas a la globalización. España: Paidós.

- CASTELLS, Manuel. “Globalización, sociedad y política en la Era de la Información”. En: Revista Análisis Político IEPRI, Universidad Nacional. No 37, mayo-agosto de 1999.

- GARCÍA Canclini, Néstor (1995). Consumidores y ciudadanos. Conflictos

culturales de la globalización. México: Editorial Grijalbo.

- LiNS Ribeiro, Gustavo (2003). Postimperialismo. Cultura y política en el mundo contemporáneo. Barcelona: Gedisa editores.

- MARÍN, Martha y MUÑOZ, Germán (2002). Secretos de mutantes. Música y creación en las culturas juveniles. Bogotá: Siglo del Hombre Editores, Universidad Central - DIUC. 
- McCHESNEY, Robert W. (2003). The New Global Media. En: HELD, David y McGrew, Anthony. The Global Tranformations Reader. An Introduction to the Globalization Debate. Cambridge: Polity Press, Blackwelll Publishing.

- PEREZ DE CUELLAR, Javier (1996). Our Creative Diversity. Report of the World Commission on Culture and Development. Paris: Unesco.

- POSEY, D.A. and DUTFIELD, G. (1996). Beyond Intellectual Property: Towards Traditional Resource Rights for Indigenous Peoples and Local Communities. Ottawa: International Development Research Centre.

- REY, Germán (1998). Balsas y Medusas. En: Visibilidad comunicativa y narrativas políticas. Bogotá: CEREC, Fundación Social, Fescol.

- SASSEN, Saskia (1996). La ville globale. Paris: Descartes y Cie.

- SCHOTT, Jeffrey J. (Ed) (2004). Free Trade Agreements. US Strategies and Priorities. Washington D.C.: Institute for International Economics.
- SMIERS, Joost (2002). The Abolition of Copyrights: Better for Artists, Third World Countries and the Public Domain). En: TOWSE, Ruth. Copyright in the Cultural Industries. London: Cheltenham.

- SMIERS, Joost (2003). Arts Under Pressure. Promoting Cultural Diversity in the Age of Globalization. Londres: Zed Books.

- TOMLinson, John (1999). Globalization and Culture. Cambridge: Polity Press.

- TOMLinson, John (2003). Globalization and Cultural Identity. En: HELD, David y McGREW, Anthony. The Global Tranformations Reader. An introduction to the Globalization Debate. Cambridge: Polity Press, Blackwelll Publishing.

- THOMPSON, John B. (1998). Los Media y la Modernidad. Una teoría de los medios de comunicación. Barcelona: Paidós. 